\title{
Éléments de morphologie quotidienne Contribution à l'étude des conditionnements de l'épenthèse présuffixale
}

\author{
Plénat*, Marc, \& Roché**, Michel \\ *ERSS (CH) / CLVA - plenat@univ-tlse2.fr \\ **ERSS (CH) / Université Toulouse-Le Mirail - mroche@univ-tlse2.fr
}

\begin{abstract}
Sauf erreur de notre part, il n'existe pas d'étude systématique du conditionnement de l'épenthèse consonantique devant suffixe dans les mots nouvellement forgés en français actuel. On ne trouve que des études qui, portant sur l'ensemble du vocabulaire en usage, mettent sur un même pied des formes très anciennes et des formes contemporaines et ne prennent en compte qu'un faible échantillon de ces dernières (cf. notamment Nyrop 1908, Pichon 1942, Stein 1971, Pagliano 2003, 2005). Comme nous avions réuni d'une façon assez systématique les dérivés formés actuellement sur les noms des jours de la semaine, et que parmi ces dérivés les épenthèses foisonnent, nous avons décidé d'étudier dans le corpus collecté les contraintes qui pèsent sur le choix de la consonne - ou parfois de la séquence - insérée entre lundi, mardi, etc., et les suffixes nominaux et adjectivaux qui les suivent. Notre étude ne prétend pas donner une description d'ensemble de l'épenthèse en français contemporain, mais elle révèle une grande diversité dans les choix opérés par les locuteurs et nous conduit à mettre l'accent sur l'aspect lexical du conditionnement de ces choix.
\end{abstract}

Après avoir - brièvement - décrit les dérivés formés sur les noms de jour et - guère plus longuement rappelé ce que l'on croit savoir de l'épenthèse, nous donnerons une idée des données que nous avons rassemblées sur l'épenthèse dans ces formations, et nous montrerons que le choix de la consonne insérée peut dépendre en particulier de la fréquence dans le lexique des différentes consonnes devant le suffixe considéré, de la catégorie syntaxique du dérivé quand le suffixe sert à former à la fois des adjectifs et des noms, et, le cas échéant, de la série sémantique à laquelle il s'agrège.

\section{Préliminaires 1 : les dérivés de noms de jours}

Dans les dictionnaires, les dérivés de noms de jour de la semaine se comptent pour ainsi dire sur les doigts de la main : dominical, mercuriale, endimancher; dimanchier (Littré) ; lundiste (Littré, TLF); dimanchard, dimanchement, lundiser (TLF). Si, dans cette petite liste, les dérivés de DIMANCHE sont de loin les plus nombreux, c'est à l'évidence que le dimanche se distingue des jours de semaine: traditionnellement, c'était le seul jour chômé, voué au culte divin et aux rites familiaux (repos dominical, messe et repas dominicaux), pour lequel les classes modestes s'habillaient « en dimanche » (endimancher, dimanchement), et envahissaient les lieux publics de leur présence bruyante importune aux nantis (dimanchier / dimanchard). Pour ceux-ci, en revanche, chaque jour de semaine pouvait échapper à la monotonie de la vie «de tous les jours ». Les dictionnaires ne consacrent pas d'entrées spécifiques aux métonymies, mais ils ne manquent pas d'indiquer que chaque jour de la semaine pouvait donner son nom aux réunions, réceptions, conférences ou spectacles organisés par tel ou tel notable (cf. les mardis de la marquise de Lambert, ou, plus tard, de Mallarmé). Ou à telle ou telle chronique hebdomadaire (cf. les lundis de Sainte-Beuve, le lundiste qui a tant lundisé).

L'individuation des jours de semaine n'est cependant pas le privilège des seules classes oisives : chacun d'entre eux a sa couleur particulière pour l'ensemble de la population ou pour certaines de ses composantes : le lundi est le jour redouté et haï de la reprise du travail (sauf à célébrer la saint lundi); le jeudi était naguère le jour de congé des écoliers, il est aujourd'hui remplacé dans ce rôle par le mercredi ; le vendredi, qui était un jour néfaste, où l'Église imposait l'abstinence, est marqué désormais par 
l'impatiente attente du week-end; c'est aussi le jour de la prière pour les musulmans; enfin, jour du Shabat (auquel il doit son nom), le samedi est surtout maintenant le premier jour du week-end, consacré souvent à des activités extra-professionnelles. Il n'est guère que le mardi qui soit quotidien. Cette spécificité de chacune des journées légitime la création de dérivés de noms de jour et ne va pas sans peser sur la productivité des différents types de dérivés ${ }^{1}$.

On trouve sur la Toile une profusion de dérivés différents des différents noms de jours. Certains locuteurs s'essaient à retrouver les schèmes anciens à l'origine de dominical et de mercuriale et inclinent à parler de leurs activités martiales (du mardi), joviales (du jeudi), vénériennes (du vendredi) ou même saturnales (du saturdi). Mais ce ne sont là que des curiosités. La plupart des dérivés sont formés sur les thèmes français lundi, mardi, mercredi, etc.

Tous les types de rapport syntaxique entre base et dérivé sont attestés :

- $\mathrm{N} \rightarrow \mathrm{N}$ :

- évaluatifs : mercredinet, lundoche, mardoche, etc., lundiche, mardiche, etc., lunduche, mercreduche, etc., lundouille, mardouille, etc., lundaille, mardaille, etc. - très fréquents depuis le retour du jeudaille! -, vendredingue, samedingue ;

- anthroponymes, par simple métonymie : Vendredi et son féminin Vendredite, etc., ou par suffixation : Lundinet(te), Mardinet(te), etc. ;

○ noms d'agent : mardiste, mercredier, jeudiard, samedinet(te) ;

- noms d'événement, métonymiques, cf. les Mardis de l'ESSEC, ou suffixés : mercrediade, jeudinette ;

- noms de disposition subjective ou objective : lunditude, etc., lundisme, etc., lundite, etc., lundiose, etc. ;

- noms de qualité : lundité, mardité ;

$\circ \quad$ et peut-être noms d'objets : dimanchette;

- $\quad \mathrm{N} \rightarrow \mathrm{V}:$

- par conversion : lunditer, lundir, etc.;

- par suffixation : lundifier, lundiser, etc.;

- ou par préfixation : enjeudier ;

- $\mathrm{N} \rightarrow$ Adj.: surtout des adjectifs de relation: lundiaire, mardiesque, mercredier, jeudial, vendredique, samedien, etc. ;

- $\quad \mathrm{N} \rightarrow$ Adv. : lundiment, mardiment, etc.

Du point de vue sémantique, on note en particulier que, noms de temps, les noms de jour de la semaine entrent aisément en tant que circonstants temporels dans la représentation de l'action ou de l'état à laquelle renvoient leurs dérivés : la lunditude est l'état d'esprit dans lequel on se trouve le lundi, une jeudinette peut être un événement modeste ayant lieu le jeudi, un vendrediste un agent se livrant à son activité le vendredi. Il n'est pas jusqu'aux lieux qui ne puissent être dénommés à partir du jour où ils servent de cadre à une activité hebdomadaire. Pérec (Espèce d'espaces, pp. 45-46) imaginait

«des appartements de sept pièces, respectivement appelées: le lundoir, le mardoir, le mercredoir, le jeudoir, le vendredoir, le samedoir et le dimanchoir. »

Ces créations n'ont pas le même statut que les mots présents dans le lexique mémorisé. Les locuteurs qui ne laissent pas tristement inemployée leur aptitude de locuteur natif à créer des mots nouveaux ont tout à fait conscience de leurs transgressions du pacte implicite restreignant l'emploi des mots à celui-ci. Ils ne 
se font pas faute, très souvent, de souligner ce caractère transgressif en modalisant d'une façon ou d'une autre leur énoncé :

En vitesse... j'vous fait part de mon bonheur mercredical (ça ce dit pas je sais!!) paskou.skyrock.com/12.html

Zhom n'était pas encore rentré de son traditionnel repas «mercredical» (mais si, ça existe!! mdr ;-))

blog.aufeminin.com/blog/seeone.../Dure-soiree-que-celle-d-hier

sébastien brault dans une session de mercredi (mercredicale?) où j'ai pris quelques photos databmx.com/forum-bmx/topic-423-1.html

Mais ce n'est pas là, à nos yeux du moins, une raison suffisante pour s'abstenir d'étudier ces créations. Au contraire, ce n'est qu'ainsi que l'on peut saisir in vivo le jeu des forces qui imposent aux mots leur forme et leur sens. Le lexique mémorisé est très opaque de ce point de vue : forme et sens y sont imposés par la force de l'habitude, au terme parfois de longues et profondes évolutions (cf. dominical et mercuriale). Ce n'est pas à dire que le morphologue doive faire abstraction des mots communs, qui servent de modèle aux mots nouveaux. Mais les mots peuvent survivre très longtemps à l'état de langue qui les a vu naître et qui explique leur genèse.

\section{Préliminaires 2 : l'épenthèse pré-suffixale}

La présente communication s'appuie sur l'idée que la morphologie dite «constructionnelle » est essentiellement une morphologie lexicale : le lexique tel qu'il naît émane du lexique tel qu'il est (cf. Roché 2009). Dans cette perspective, on est amené à admettre que le conditionnement des allomorphies radicales est pluriel : il repose certes pour une part sur des contraintes phonologiques générales (peut-être même universelles), mais la forme que prennent les mots nouveaux résulte aussi de généralisations globales ou locales sur le lexique mémorisé (cf. Plénat 2009). L'épenthèse présuffixale fournit à notre sens de bons arguments en faveur de cette approche.

Pour illustrer notre propos, nous prendrons pour exemple les dérivés en -esque de vendredi que nous a fournis une exploration de la Toile :

vendredi-esque (10 occurrences)

Ex. : Le problème c'est que même si le resultat est tout à fait convainquant, vos amis risquent de suprendre votre petit jeu de fainéantise vendredi-esque.

www.ciao.fr > ... > Plats cuisinés exotiques, spécialités étrangères

vendredesque (49 occurrences)

Ex. : Merci et salutations vendredesques a vouzaussi :-)

fr.twitter.com/Tamala 75

vendrediesque (123 occurrences)

Ex. : moi qui avait fui l'agitation BUienne du vendredi, je vais devoir y retourner car visiblement, je suis atteinte d'une improductivite vendrediesque chronique. superdoublantep6.canalblog.com > Messages octobre 2009

vendreditesque (16 occurrences)

Ex. : Bises vendreditesques.

lechaudrondetaka.canalblog.com/archives/2011/03/.../20536979.htm...

vendredinesque ( 7 occurrences)

Ex. : Coucou vendredinesque ma CojCop.

fr.answers.yahoo.com > ... > Toutes les catégories > Éducation > Futilités 
vendredilesque ( 1 occurrence)

Ceci était un message vendredilesque.

forums.mondespersistants.com > ... > [MGW] Discussions générales

vendredillesque ( 1 occurrence)

Heu... sans doute beauuuuuuuuucoup trop pour moi (gratuit c'est déjà trop cher); c'était juste pour le clin d'oeil vendredillesque sinon c'est vrai, c'est emmerd... quand ça tombe en rade. forum.hardware.fr/.../techniques-abandonnees-appareils-sujet_67751...

vendredidesque (14 occurrences)

Ex. : Comment comment ! je ne sens pas la liesse vendredidesque ce matin :yahoo: www.forumfr.com/sujet393815-post590-le-club-des-leve-tot-du-foru...

vendredisesque (1 occurrence)

Bisous vendredizesques...

leonor.unblog. fr/2006/10/05/11-minutes-vii/comment-page-2/

Considéré seul, ce petit corpus autoriserait un traitement purement phonologique des deux principaux problèmes que soulève l'épenthèse présuffixale en français, l'apparition d'une consonne intercalaire et le choix de la consonne intercalée. Mais ce que l'on sait par ailleurs de ces phénomènes interdit de s'arrêter à ce type d'explication.

Commençons par le conditionnement de l'épenthèse elle-même. Comme c'est le plus souvent le cas, les consonnes épenthétiques des dérivés en -esque de vendredi sont insérées entre une séquence s'achevant par une voyelle et un suffixe commençant également par une voyelle. Dans ce type de contexte, l'épenthèse n'est pas le seul phénomène observable : s'il arrive que les deux voyelles restent en hiatus (cf. les graphies comme vendredi-esque), il n'est pas rare que la première voyelle disparaisse (cf. vendredesque), ou qu'elle soit semi-vocalisée (il est probable que la graphie vendrediesque correspond dans un certain nombre de cas à une prononciation où le $i$ note un yod). La troncation et la semivocalisation aboutissant, comme l'épenthèse, à une prononciation sans hiatus, cette gamme de réactions invite à recourir à l'une ou l'autre des versions modernes de la classique «horreur de l'hiatus ». Dans la Théorie de l'Optimalité (cf. Prince \& Smolensky 1993, McCarthy \& Prince 1993) par exemple, on pose ainsi une contrainte ATTAQUE voulant que, toutes choses égales d'ailleurs, il soit préférable qu'une syllabe commence par une attaque consonantique. Cette contrainte anti-marque se heurte à diverses contraintes de fidélité exigeant qu'à tout élément de la base corresponde un élément du dérivé (interdiction d'effacer), de préférence un élément identique à celui-ci (interdiction, par conséquent, de semi-vocaliser), et que tout élément du dérivé corresponde à un élément de la base (interdiction d'insérer). Variables de locuteur à locuteur, les rapports de force qui s'instaurent entre ces contraintes suffisent à expliquer la gamme des réactions. Dans ce cadre, les formes à épenthèse résultent de la prééminence de la contrainte Attaque et de la faiblesse de la contrainte interdisant les insertions.

Toutefois, cette explication par l'horreur de l'hiatus paraît un peu courte dans le cas du français. Des études comme celles de Stein (1971) ou de Pagliano (2003) ne manquent pas de relever que, si l'on trouve des consonnes intercalaires devant les suffixes, on n'en trouve pas derrière les préfixes (cf. antiesquimaux, antiespagnol). Cette dissymétrie interdit de penser que le conditionnement de l'épenthèse soit purement phonologique. Pagliano (ibid.) suppose que l'explication est à chercher du côté du suffixe : certains d'entre eux fourniraient un site consonantique propre à accueillir la consonne épenthétique ${ }^{2}$. Nos données suggèrent qu'en fait l'épenthèse n'est pas l'apanage de suffixes particuliers et montrent que les suffixes qui l'admettent tolèrent aussi des formes sans consonne intercalaire : le suffixe -esque n'est pas un cas isolé. Dans ces conditions, il est sans doute plus sage de chercher une explication du côté de la base plutôt que du suffixe et d'attribuer le recours aux consonnes intercalaires à «la difficulté de déterminer ou d'imaginer une forme de liaison à certains substantifs nominaux à terminaison vocalique orale nue " (Pichon, 1942 : 28). En termes plus modernes, on peut imputer l'apparition de ces consonnes au fait que le thème employé devant suffixe - le thème $B$ des nominaux dans Roché (2010) - s'achève ordinairement par une consonne, que le thème employé dans la forme libre - le thème $\mathrm{A}$ des nominaux de Roché (2010) - soit pourvu ou dépourvu d'une telle finale. Ne valant évidemment pas pour les préfixes, cette contrainte 
globale sur la forme des thèmes liés des nominaux permet d'expliquer la dissymétrie observée entre la joncture préfixale et la joncture suffixale.

Cette hypothèse a en outre pour elle le fait que des consonnes très analogues aux consonnes intercalaires présuffixales sont très souvent insérées à la fin des nominaux masculins s'achevant par une voyelle pour marquer le féminin. Les masculins vendredi et Vendredi donnent ainsi les féminins vendredite et Vendredite (ou Vendredine) désignant par métonymie une chronique paraissant le vendredi ou la compagne d'un Robinson :

Mais je vais me faire pardonner dans cette chronique du vendredi, que je vais intituler « Les vendredites ».

la-vie-lunivers-et-tout-le-reste.blogspot.com/.../les-vendredites.html

Ben ouais, une île déserte c'est une île déserte... même avec "Vendredite" pour combler tes siestes crapuleuses www.princepassion.com/tag/passion/

Robinsonne Et Vendredine / Aubryet Xavier (Auteur) www.chapitre.com/.../fr/.../robinsonne-et-vendredine,28892380.aspx

Comme le féminin des nominaux recourt également au thème $\mathrm{B}$ des masculins, ces consonnes trouvent elles aussi une explication dans l'idée que ces thèmes tendent à s'aligner sur le schème prédominant à consonne finale.

Les discussions sur l'épenthèse présuffixale en français ne manquent pas non plus de relever qu'on trouve parfois des consonnes intercalaires après consonne. L'exemple le plus communément cité est celui des thèmes $\mathrm{A}$ en /ar/ qui donnent des thèmes $\mathrm{B}$ en /ard/ même quand l'étymologie semble devoir l'interdire. On trouve par exemple sur la Toile une quantité non négligeable de aldomovardesque, de bazardesque, de bizardesque, de hangardesque et de tintamardesque à côté des formes plus correctes aldomovaresque, bazaresque, bizarresque, hangaresque et tintamarresque. L'horreur de l'hiatus est, dans ce cas aussi, impuissante à rendre compte de l'épenthèse. C'est assurément une généralisation locale qui explique le phénomène : le thème $\mathrm{B}$ de bazar, bizarre, etc. s'aligne sur les nombreux thèmes $\mathrm{B}$ en /ard/. Les féminins des adjectifs en /ar/ comportent eux aussi à l'occasion un /d/ superfétatoire : Frei (1929: 47) cite ainsi les formes « fautives » avarde, bizarde, hilarde et ignarde. Ce parallélisme entre dérivés et formes féminines confirme l'idée que les consonnes épenthétiques présuffixales doivent être traitées dans la rubrique de la formation des thèmes.

Dans le cas du /d/ de bizardesque ou de bizarde, c'est la séquence qui précède la consonne intercalaire qui impose la présence de celle-ci. Il existe aussi des cas, probablement moins connus, où c'est la séquence qui suit la consonne qui impose sa présence ${ }^{3}$. Mir Marashi (2005) signale ainsi l'existence de dérivés en -titude construits sur des bases à finale consonantique : black donne à la fois blackitude et blacktitude, geek : geekitude et geektitude, plouc: plouquitude et plouctitude, cheap : cheapitude et cheaptitude, rap : rapitude et raptitude, salope : salopitude et saloptitude. Nul doute que le /t/ intercalé ne résulte dans ce cas d'un alignement sur les très nombreux dérivés en -itude construits sur des radicaux en /-t/ figurant dans le lexique mémorisé (béatitude, platitude, exactitude, aptitude, etc.).

Nous sommes ainsi conduits à imputer l'apparition des consonnes intercalaires présuffixales à l'existence de schèmes résultant de généralisations de très inégale ampleur pouvant porter tant sur des séquences de phonèmes particulières que sur la forme générale de certains thèmes plutôt qu'à l'« horreur de l'hiatus ». Cela ne veut pas dire que celle-ci soit un mythe ni qu'elle ne joue aucun rôle dans l'affaire. Pichon (loc. cit.) suggère que la contrainte poussant le locuteur à imaginer une «forme de liaison » devant le suffixe « s'ajout[e] à une certaine répugnance à l'hiatus ». Sa vision cumulative des contraintes est probablement la bonne. Mais toujours est-il que cette répugnance n'explique pas à elle seule l'épenthèse et n'est même pas déterminante. 
Le conditionnement du choix des consonnes épenthétiques présuffixales se laisse lui aussi apparemment décrire à l'aide de contraintes phonologiques. La typologie nous apprend que la consonne intercalaire peut avoir deux types de provenance : elle peut répéter un phonème figurant dans l'environnement proche, ou devoir sa présence à son caractère non marqué (cf. Kitto et Lacy 1999). Les deux types de consonne figurent dans l'inventaire des dérivés en -esque de vendredi. Dans vendredillesque (et probablement dans un certain nombre de réalisations de vendrediesque avec un «yod de transition »), la consonne épenthétique est une réplique non syllabique de la voyelle finale de vendredi; dans vendredidesque, il y a toutes chances que le /d/ épenthétique soit emprunté à la base; il est possible enfin que le /z/ de vendredisesque soit une version voisée $\mathrm{du} / \mathrm{s} / \mathrm{du}$ suffixe, mais l'attestation unique de ce dérivé interdit qu'on épilogue sur ce cas. Parmi les autres dérivés à épenthèse de vendredi, le / $\mathrm{t} / \mathrm{de}$ vendreditesque est certainement, comme on le verra, le meilleur candidat au statut de consonne non marquée, mais on ne saurait manquer de remarquer que l'ensemble des consonnes intercalaires appartiennent à la classe des coronales.

Nous renvoyons à Kitto \& Lacy (art. cit.) pour une explication détaillée de l'épenthèse réduplicative dans le cadre de la Théorie de l'Optimalité. Très grossièrement ce type d'épenthèse trouve sans doute son explication dans l'idée que tout phonème d'une forme donnée doit correspondre en principe à un phonème de la forme dont cette forme est issue. En l'absence de toute consonne finale dans la base, le dérivé va chercher la consonne qui lui manque dans le radical ou dans le suffixe. Au mépris de la contrainte voulant qu'à un phonème de la forme-mère ne corresponde qu'un phonème dans le forme-fille, et des contraintes dissimilatives.

Il nous faut cependant dire deux mots ici de la réduplication épenthétique d'une consonne interne qui, contrairement à l'épenthèse d'un yod après /i/, nous semble n'avoir jamais été documentée en français. Nous avons toutes raisons de croire que vendredidesque est bien un dérivé légitime. D'une part, en effet, le /d/ est l'une des consonnes intercalaires les mieux attestées dans les dérivés en -esque de vendredi (14 occurrences dans nos données); d'autre part, nous avons aussi trouvé des occurrences de vendredidal (1), vendredidette (1), vendredidien (4) et vendredidique (1). Ce qu'il y a de curieux et d'intéressant, c'est que ces épenthèses de /d/ sont beaucoup plus rares dans les dérivés des autres noms de jour de semaine ( 2 occurrences de mardidien, 2 de mercredidien, 1 seulement de lundidesque, de mardidesque et de samedidal). À notre sens, l'unique trait caractéristique de vendredi susceptible d'expliquer le fait que, seul parmi les noms de jour, ce nom semble vraiment autoriser l'épenthèse d'un /d/ réside dans le fait que ce mot comporte déjà deux /d/ : la contrainte dissimilative (cf. infra) étant déjà enfreinte une fois, il est peutêtre moins grave qu'elle le soit une seconde fois ${ }^{4}$.

Le statut des coronales a fait couler beaucoup d'encre. On a pu soutenir, non sans quelque vraisemblance, que ces phonèmes étaient non marqués en ce sens qu'ils seraient sous-spécifiés pour ce qui est du lieu d'articulation à un niveau sous-jacent (cf. Paradis \& Prunet 1991). Dans cette hypothèse, les consonnes épenthétiques non réduplicatives n'acquerraient que tardivement et de façon automatique leur lieu d'articulation coronal. Ce point de vue, toutefois, rencontre des difficultés. D'une part, nombre d'arguments plaident contre l'idée que les coronales soient ainsi sous-spécifiées (cf. McCarthy \& Taub 1992), et, d'autre part, il semble que les glottales - qui sont beaucoup plus souvent sollicitées que les coronales comme consonnes épenthétiques dans les langues du monde - soient de meilleures candidates au titre de consonnes les moins marquées (cf. Lombardi 2002). Ce qui conduit cet auteur à adopter l'idée que le caractère plus ou moins marqué des points d'articulation découle d'une hiérarchie de caractère universel au sein de laquelle le point d'articulation coronal occuperait le second rang; de ce fait, les langues qui, comme le français, sont dépourvues de glottales seraient amenées à utiliser les coronales comme consonnes épenthétiques.

Les listes de consonnes épenthétiques fournies par les auteurs qui se sont intéressés à la question en français (e.g. Nyrop op. cit: 54-61, Pichon, op. cit: 25-36, Stein, art. cit.: 57-61, Porquier 1993) comportent presque exclusivement des coronales. Mais les auteurs signalent aussi que, parmi les coronales, la consonne adventice qui prédomine, et de loin, est le /t/, dont on peut saisir au vol d'innombrables exemples. Ce qui conduit Pichon (op. cit : 33) à poser que « [1]e phonotype [t] s'est constitué dans le français d'aujourd'hui une province fonctionnelle où il règne en maître : le rôle de 
consonne intercalaire. ». Comment expliquer cette prédominance ? Et comment expliquer que d'autres coronales apparaissent?

La prédominance de /t/ peut avoir des raisons phonologiques : en tant qu'occlusive sourde c'est lui qui, parmi les coronales, assure la meilleure courbe de sonorité à la syllabe dont la consonne intercalaire occupe l'attaque (cf. Lombardi, art. cit.). Le choix d'une autre consonne trouve probablement souvent aussi une explication dans la phonologie : les éléments interfixés - consonnes intercalaires et interfixes en $\mathrm{VC}$ - sont très sensibles aux contraintes dissimilatives. Il a été montré (Plénat, à paraître), par exemple, que la consonne /t/ était extrêmement peu souvent intercalée devant le suffixe -ité, et (Plénat 2005) que les base s'achevant par un /t/ ou un /d/ n'admettent guère que des interfixes en sonante (surtout -el-, -in- et -oun-) devant le suffixe -ette. On ne s'étonne donc pas trop que vendredi, dont la dernière attaque est l'occlusive coronale /d/ puisse donner, à côté de vendreditesque, tantôt vendredinesque et tantôt même parfois vendredilesque. Comme souvent, l'issue du conflit entre contraintes est incertaine.

Il est ainsi très souvent concevable de rendre compte du choix de la consonne épenthétique en termes purement phonologiques. Mais il est clair aussi que cela n'est pas toujours possible. Les études classiques (par exemple Nyrop, op. cit., Stein, art. cit.) mettent en avant l'idée que la consonne choisie permet dans nombre de cas d'aligner la finale du radical sur une finale fréquente. De fait, il serait difficile, par exemple, de ne pas supposer que le choix qui est fait de /d/ après /ar/ n'a rien à voir avec le suffixe péjoratif /-ard/ : bizarde et bizardesque s'alignent sur couarde, couardise, nullarde, nullardise, etc. Les cas d'épenthèse qui se laissent analyser de cette façon sont très nombreux. L'alternance -eux -euse que l'on a dans gueux / gueuse, gueuserie, heureux / heureuse, heureusement, etc. permet d'expliquer l'apparition d'un /z/ dans banlieusard ou bondieuserie, l'alternance -aud -aude que l'on a dans chaud / chaude, chaudement ou nigaud / nigaude, nigauderie se retrouve dans bedeau / bedeaude, bedeaudaille, boyau / boyaudier, etc., et ainsi de suite. D'autres études, moins connues, signalent d'autres phénomènes. Il a été suggéré que les consonnes intercalaires peuvent avoir aussi pour origine :

1. des finales de sous-séries particulièrement bien représentées dans une série suffixale. C'est le cas, par exemple du /r/ dans les dérivés en -aie (Roché 2011): acaciaraie, hévéaraie, thuyaraie, kiwiraie, caféraie, cacaoraie... Si l'épenthèse en /r/ est la plus fréquente devant ce suffixe, c'est que par ailleurs les finales en -eraie sont particulièrement nombreuses dans cette série lexicale à cause du grand nombre de bases en -ier (le type palmier palmeraie). C'est aussi, rappelons-le, le cas du /t/ que l'on trouve si souvent devant -itude dans les formations nouvelles, même après consonne, malgré l'entorse aux contraintes dissimilatives : béat-itude, prompt-itude, exact-itude amènent neuneu-t-itude, gaga-t-itude, moch-t-itude, plouc-t-itude (cf. Mir Marashi, op. cit.).

2. des consonnes empruntées à un autre dérivé de la base. Pichon (op. cit., p. 30) suggère que le /1/ de hugo-l-ien provient de hugo-lâtre. Ce n'est pas le seul exemple de ce genre. Nous avons par exemple dans nos données des manga-qu-esque et des manga-qu-este 'relatif aux mangas' qui ne s'expliquent guère que par référence à manga-ka, des pacha-l-esque 'digne d'un pacha' qui pourraient trouver une explication dans pacha-lik, et un statu-f-ien qui doit à coup sûr son /f/ à statu-fier.

3. des lexèmes sans lien de parenté autre que phonétiques avec la base. S'il arrive à bambou de donner bamboulesque, à saga de faire saganien et à Astra d'avoir pour dérivé astraqueste, c'est probablement parce que bambou est déjà suivi d'un $l$ dans bamboula, saga d'un $n$ dans Sagan et astra suivi d'un $k$ dans astrakan (Plénat 2009).

Ce rapide examen de l'épenthèse présuffixale en français montre que le conditionnement de ce phénomène est complexe, qu'il comporte à la fois des aspects purement phonologiques et des aspects morphologiques et lexicaux, des schèmes et des motifs récurrents ou saillants imposant parfois la présence d'une consonne ou même le choix d'une consonne déterminée devant le suffixe. Le cadre théorique qui se prête le mieux à l'explication de cette complexité nous paraît être celui que développe Burzio (cf. notamment Burzio 2001), dans lequel chaque élément d'une représentation implique tous les autres et où les implications co-présentes dans un lexème ou récurrentes dans le lexique cumulent leurs 
forces. Dans ce cadre, on peut comprendre à la fois que le grand nombre des thèmes B à consonne finale induise souvent la présence d'une telle consonne quand le lexique n'en fournit pas déjà une et qu'un lexème individuel comme bamboula impose le choix de /1/ comme consonne intercalaire dans le dérivé en -esque de bambou. Ce genre de contraintes lexicales interférant avec les contraintes phonologiques proprement dites, on comprend aussi que la phonologie impose parfois ses propres solutions, comme dans le cas de l'épenthèse réduplicative et parfois cède à la pression du lexique, comme dans les cas d'épenthèse post-consonantique.

Le socle empirique sur lequel repose l'étude des épenthèses en français est relativement réduit. Pagliano $(2003,2005)$ se fonde sur une liste de moins de 300 cas. Les exemples sur lesquels nous nous sommes nous-mêmes fondés jusqu'ici pour illustrer la complexité de leur conditionnement sont épars et peu nombreux. Il était donc utile d'étudier systématiquement les consonnes intercalaires apparaissant devant suffixe dans les dérivés des noms de jour de semaine.

\section{Le corpus}

Nous avons établi la liste des consonnes apparaissant entre les noms de jour de semaine et une dizaine de suffixes en interrogeant systématiquement Google en français (oct. 2011) ${ }^{5}$. C'est un travail long et fastidieux, mais nous n'avions pas d'autre moyen de procéder, puisque les moteurs de recherche n'admettent plus l'emploi des expressions régulières et luttent contre celui des robots. Le caractère systématique de la récolte permet de constituer des données sans biais théorique. Les résultats ont confirmé beaucoup de nos intuitions, mais nous avons aussi repéré des configurations dont nous n'aurions jamais imaginé qu'elles pussent exister.

Toutes les consonnes n'apparaissent pas dans la position examinée et celles qui apparaissent apparaissent en quantité très variable. Nous n'avons pas été en mesure de déterminer le nombre des attestations de chaque consonne devant chaque suffixe, nous avons dû nous contenter de noter l'existence ou l'absence de telles attestations. On trouvera dans le tableau 1 le nombre des jours de semaine pour lesquels chaque consonne est attestée devant chaque suffixe :

\begin{tabular}{|c|c|c|c|c|c|c|c|c|c|c|c|c|c|c|c|c|c|c|}
\hline & $\mathrm{t}$ & d & S & z & $n$ & 1 & $\mathrm{r}$ & $p$ & $b$ & $\mathrm{f}$ & $\mathrm{v}$ & $\mathrm{m}$ & $\mathrm{k}$ & g & $\int$ & 3 & $\mathrm{n}$ & $\mathrm{j}$ \\
\hline -al & 6 & 2 & & & 6 & & & & & & & 1 & 6 & 1 & & & & \\
\hline -esque & 6 & 3 & 1 & 1 & 6 & 4 & 1 & & & & & & 1 & & 1 & & & 1 \\
\hline -ette & & 1 & & 6 & 6 & & & 5 & & & & & & & & & & \\
\hline -iaque & & & & 5 & & & & & & & & & & & & & & \\
\hline -ien & & 3 & 6 & 6 & 6 & 1 & 3 & & 1 & & & & & & & & & \\
\hline -aire & 1 & & & & 2 & & & & & & & & & & & & & \\
\hline -eux & 4 & & & 2 & 4 & & & & & & & & & & 1 & & & \\
\hline -ier & 4 & & 2 & 1 & 2 & & & & & & & & & & & & & \\
\hline -ique & 1 & 1 & & & 1 & & & & & & & & & & & & & \\
\hline
\end{tabular}

Tableau 1 : Consonnes épenthétiques dans les dérivés de noms de jour de semaine 
Ainsi par exemple le chiffre 3 au croisement de la ligne -esque et de la colonne /d/ signifie qu'une épenthèse en $/ \mathrm{d} /$ est attestée pour les dérivés en -esque de trois noms de jours différents. Pour une meilleure lisibilité, nous avons proportionné le niveau de gris des cellules au nombre qui y figure.

Comme le laissaient prévoir les études portant sur l'épenthèse en français, la plupart des consonnes repérées sont des coronales, occlusives $(/ \mathrm{t} /, / \mathrm{d} /, / \mathrm{n} /)$ ou fricatives $(/ \mathrm{s} /, / \mathrm{z} /)$. Il y a sans nul doute une part de vérité dans l'idée que l'articulation dentale est l'articulation non marquée et que les consonnes épenthétiques doivent être, de préférence, non marquées quant au lieu d'articulation. Cependant, la finale $/ \mathrm{kal} /$ est présente - en abondance - après les six jours de semaine, et la finale /pet/ - chez un seul locuteur et en très faible quantité - après cinq d'entre eux. En dehors de ces deux cas, on n'a affaire qu'à une poussière d'exceptions probablement fautives ou anecdotiques, à des jeux de mot (jeudirien, samedirien = 'je dis rien', 'ça me dit rien', samedibien = 'ça me dit bien'), ou bien encore à des cas où la consonne peut provenir de la base (mardichesque peut être construit sur mardiche, diminutif assez courant de mardi).

Parmi les consonnes dentales, ce n'est pas le /t/ qui prédomine, mais le /n/. Celui-ci n'est absent que devant -IAQUE et il est attesté pour chacun des six jours ouvrables devant 4 des 9 suffixes étudiés (-AL, ESQUE, -ETTE, -IEN), alors que / $t$ / est absent devant trois suffixes et n'est attesté après les noms des six jours de semaine que devant -AL et -ESQUE. /d/ est attesté, mais en quantité très restreinte, devant 5 suffixes sur 9 ; nous avons vu ci-dessus qu'il n'était en fait bien attesté qu'après vendredi. Après les occlusives nasales et orales, ce sont les fricatives qui sont les mieux représentées. Plusieurs cas sont à distinguer: les dérivés en -eux ont des chances d'être des variantes populaires et plaisantes de noms d'agent en -eur (vendrediseux dans « coucou les vendrediseux », www.atoute.org > Forum > Conférence Principale, Forum Blabla, semble dire la même chose que vendrediseur dans «arrivée des vendrediseurs », 500-xt-le-gros-mono.bbflash.net/t4963-we-xt-sr-2009-planninje); la sifflante, dans leur cas, pourrait être celle du suffixe -is(er). Les très peu nombreuses sifflantes qui apparaissent (curieusement!) devant -esque peuvent être des épenthèses réduplicatives. En dehors de cette poussière de cas marginaux, il faut distinguer deux cas de figure principaux. Les sifflantes concurrencent la nasale dans deux contextes où le $/ \mathrm{t} / \mathrm{est}$ proscrit : devant - ette $e^{6}$, et devant -ien (nous supposons que devant cette dernière finale la lettre $t$ est prononcée comme une sifflante). Le cas des dérivés en -iaque est différent : devant cette finale, seul le /z/ est attesté, et sa présence semble nécessaire (pas de dérivés comme lundiaque, mardiaque, etc). On observe cette épenthèse après cinq des six noms de jour, dans plus d'une quinzaine d'attestations de provenances diverses.

On ne peut assurément pas être pleinement satisfait des données recueillies. Il nous manque de multiples informations, tant sur les locuteurs (leur origine géographique, leur âge, leur statut social, leur degré de culture) que sur la pertinence des données recueillies (leur légitimité, la proportion de chaque type par rapport aux autres types, le type de discours dans lesquels elles apparaissent). On constate une variation considérable dans le choix des consonnes intercalaires sans pouvoir rapporter cette variation à des variables identifiées. Les données électroniques nécessiteraient de longues et minutieuses enquêtes philologiques, un investissement en travail et en temps dont les bénéfices sont incertains. On ne manquera pas, toutefois, de noter que, comparées aux données habituellement prises en compte (deux ou trois centaines de formes d'âges divers dans le meilleur des cas), nos données forment une masse considérable : nous avons relevé environ 120 dérivés à épenthèse de nos 6 noms de jour de semaine, et constaté que d'innombrables cas de figures possibles a priori n'étaient jamais attestés.

Dans ce qui suit nous n'examinerons que les dérivations suffisamment attestées pour donner prise à l'analyse. Les dérivés en -aire, en -ier, en -ique et en -eux seront laissés de côté. Notre but est de déterminer, autant que faire se peut, quelles consonnes intercalaires ont une origine lexicale. Le problème reste parfois sans solution très claire. Lorsque les contraintes phonologiques et les pressions lexicales s'exercent dans le même sens, le problème est sans véritable solution. On verra cependant qu'à côté de ces cas incertains, il en existe de relativement clairs dans lesquels un motif présent dans le lexique impose le choix d'une consonne épenthétique déterminée. Les motifs en cause peuvent être de nature exclusivement phonologique : telle séquence de phonème peut s'imposer comme modèle du fait de sa fréquence ou de sa longueur, mais des facteurs prosodiques entrent aussi sans doute en ligne de compte. À ces facteurs phonologiques, peuvent se conjoindre des facteurs syntaxiques : la catégorie du dérivé joue à l'occasion 
un rôle ; et des facteurs sémantiques : un lexème de sens proche impose parfois sa consonne - ou même sa séquence de phonèmes - présuffixales. Comme, dans tous les cas, le - ou les - modèles appartiennent à une série suffixale, nous parlerons dans tous les cas de sous-séries.

\section{Les sous-séries : aspects phonologiques}

Il existe une corrélation assez étroite entre le choix des consonnes épenthétiques devant les différents suffixes et les consonnes apparaissant devant ces suffixes (ou des finales homophones) dans le lexique. Les consonnes choisies pour l'épenthèse sont, en règle générale, celles qui apparaissent le plus fréquemment devant la rime considérée : les dérivés de noms de jour de la semaine tendent à entrer dans des sous-séries rimiques déjà bien constituées et à conforter celles-ci en rimant richement avec leurs membres.

Nous avons relevé dans la base de données lexicales en ligne Lexique 3, qui comprend 55.000 lemmes et leur fréquence dans un corpus de textes écrits entre 1950 et 2000 tirés de Frantext et dans un corpus de sous-titres de films, le nombre d'occurrences de chaque attaque simple devant les finales des adjectifs en -al, -esque et -iaque, des noms en -ette et des noms et adjectifs en -ien (les dérivés de noms de jours en -ien ont à la fois des emplois adjectivaux et des emplois substantivaux). Ces décomptes sont résumés dans le tableau 2, où, par exemple, le chiffre 8 au croisement de la ligne -iaque et de la colonne /n/ indique que nous avons relevé 8 adjectifs s'achevant par -niaque dans la base de donnée :

\begin{tabular}{|c|c|c|c|c|c|c|c|c|c|c|c|c|c|c|c|c|c|c|}
\hline & $\mathrm{t}$ & d & $\mathrm{s}$ & z & $\mathrm{n}$ & 1 & $\mathrm{r}$ & $\mathrm{p}$ & b & $\mathrm{f}$ & $\mathrm{v}$ & $\mathrm{m}$ & $\mathrm{k}$ & $\mathrm{g}$ & $\int$ & 3 & $\mathrm{n}$ & $\mathrm{j}$ \\
\hline$-\mathrm{al}_{\mathrm{A}}$ & 67 & 11 & 6 & 4 & 75 & 1 & 61 & 7 & 5 & 4 & 7 & 16 & 43 & 9 & & & & 3 \\
\hline -esque $_{\mathrm{A}}$ & 8 & 2 & & & 8 & 8 & 13 & & & & & 2 & & & & & & \\
\hline- ette $_{N}$ & 2 & 12 & 28 & 22 & 68 & 51 & 31 & 25 & 7 & 5 & 4 & 10 & 34 & 6 & 25 & 7 & 3 & 20 \\
\hline i-aque ${ }_{\mathrm{A}}$ & & 2 & & 4 & 8 & 3 & 2 & & & & & & & & & 2 & & \\
\hline$-\operatorname{ien}_{\mathrm{A} / \mathrm{N}}$ & 13 & 43 & 102 & 31 & 75 & 51 & 81 & 10 & 13 & & 10 & 14 & 5 & 2 & 2 & 15 & & \\
\hline
\end{tabular}

Tableau 2 : Attaques des syllabes finales en -al, -esque, -ette, -iaque, -ien dans Lexique 3

Pour la commodité du lecteur, nous avons grisé les cellules des occlusives et fricatives dentales les plus fréquentes devant les finales étudiées dans le tableau 2. Un simple coup d'œil permettra de constater que les cellules grisées du tableau 2 et celles de la partie supérieure du tableau 1 sont assez exactement superposables. Il ressort bien de cette comparaison qu'en règle générale, les consonnes épenthétiques sont choisies parmi celles des occlusives ou des fricatives dentales qui apparaissent le plus fréquemment devant chacune des finales considérées dans cette base de données.

Cette coïncidence ne résulte sans doute pas toujours uniquement de l'attraction exercée par les finales les plus fréquentes. Si l'on trouve /t/ devant -al et -esque après les six noms de jour de semaine, ce peut être aussi parce que ce phonème est celui qui fournit l'attaque la moins marquée, et $\mathrm{si} / \mathrm{n} /$ est présent six fois aussi devant -al, -esque, -ette et -ien, ce peut être parce que l'insertion de /t/ contrevient à la contrainte dissimilative qui bannit autant que faire se peut la consécution de deux occlusives dentales. Il ne fait guère de doute que les contraintes purement phonologiques jouent un rôle dans le choix des consonnes insérées. Si les liquides /1/ et /r/ sont si peu souvent retenues, c'est probablement parce qu'elles fournissent de piètres attaques en termes de courbe de sonorité : leur fréquence devant certains suffixes laisserait attendre un emploi fréquent comme consonnes intercalaire si les pressions lexicales s'exerçaient seules. On peut constater dans le tableau 2 que la contrainte dissimilative interdisant les consécutions de deux occlusives dentales est si forte dans le cas de deux sourdes qu'elle exclut presque du lexique courant les 
finales en -tette. Rien d'inattendu donc à ce que /t/ ne soit pas utilisé comme consonne intercalaire devant le suffixe -ette. Il y a de bonnes chances pour que la répartition des consonnes intercalaires résulte dans bien des cas de l'influence conjointe des contraintes phonologiques et des pressions lexicales.

Dans certains cas, en revanche, ces dernières jouent un rôle prépondérant. Il n'y a en particulier guère de raison de penser que le / $\mathrm{t} /$ soit banni devant le suffixe -ien des dérivés de nom de jour en -ien pour des raisons purement phonologiques. Il n'est pas vrai que le /t/ s'assibile devant yod en français moderne : personne n'est tenté de prononcer bijoutier, émeutier ou muletier avec un /s/. Ce qui est vrai, c'est que si -ien est précédé d'une obstruante dentale, cette obstruante a toutes chances d'être une sifflante. Il y a donc tout lieu de penser que si /s/ et / z/ servent si souvent de consonne intercalaire dans les dérivés de nom de jour en -ien, c'est en raison de leur fréquence devant ce suffixe dans le lexique mémorisé. Dans ce cas, la consonne non marquée $(/ \mathrm{t} /)$ le cède à des consonnes fréquentes.

On dira peut-être qu'à ce compte, le /d/, qui est plus fréquent que le /z/ devant -ien, devrait fournir une bonne consonne intercalaire devant ce suffixe. En fait, elle est peut-être moins mauvaise qu'il n'y paraît. Comme on l'a vu, elle apparaît dans nos données non seulement dans vendredidien (4 occurrences), où elle peut résulter de la répétition d'un /d/ interne, mais aussi dans mardidien (2 occurrences) et dans mercredidien ( 2 occurrences) et, surtout, ces deux derniers dérivés sont, considérés ensemble, aussi bien attestés que le premier. Cette répartition est tout à fait différente de celle des /d/ intercalaires dans les autres dérivations, dans lesquelles le /d/ apparaît exclusivement ou très majoritairement après le seul vendredi. Les données sur lesquelles nous nous fondons sont trop minces pour qu'on avance l'hypothèse avec beaucoup d'assurance, mais il ne nous semble pas exclu que les lexèmes en -dien - notamment ceux, assez nombreux, qui se terminent par -idien comme méridien ou rachidien - du lexique mémorisé, exercent à l'occasion une influence sur les dérivés en -ien des noms de jour. Il paraît en particulier assez probable que quotidien, qui appartient au même champ lexical, joue un rôle dans cette profusion insolite d'épenthèses de $/ \mathrm{d} /$. Cette influence ne peut qu'être relativement faible, en raison de la proximité d'un autre /d/ dans le radical.

Dans un petit nombre de cas, la corrélation entre la consonne intercalaire et les consonnes les plus fréquentes devant le suffixe considéré est beaucoup moins étroite. En comparant les deux tableaux, on constate en particulier que le suffixe -iaque sélectionne uniformément /z/ comme consonne épenthétique alors que l'attaque la plus fréquente devant ce suffixe dans le lexique mémorisé est le /n/. Mais, en fait, il n'y a pas lieu de considérer la seule syllabe finale. Dans les quatre adjectifs en -siaque (dionysiaque, aphrodisiaque, anti-aphrodisiaque, paradisiaque) figurant dans Lexique 3, la syllabe finale est précédée de /i/ et, dans trois d'entre eux, de /di/, alors que dans aucun des huit dérivés en -niaque la syllabe finale n'est précédée de /i/, ni à plus forte raison de /di/. Il est clair que, dans ce cas, c'est la longueur des finales pareilles qui entre en ligne de compte: c'est parce qu'ils riment léoninement avec paradisiaque et aphrodisiaque (trois phonèmes communs avant le suffixe) que lundisiaque, mardisiaque, etc. sont préférés à lundiniaque, ${ }^{\circ}$ mardiniaque, etc., qui ne riment que richement avec bosniaque, insomniaque ou simoniaque (un seul phonème commun avant le suffixe). Il arrive d'ailleurs que le dérivé du nom de jour et l'adjectif paradisiaque apparaissent dans un même texte. La blogueuse qui raconte (un vendredi) s'être présentée le matin devant son miroir

espérant un mot gentil de sa part, un regard admirateur sur [son] « look vendredisiaque ». associationppa.wordpress.com/2011/.../vendredi-le-23-septembre-20...

souhaite quelques lignes plus loin à ses lectrices un « vendredi paradisiaque ».

On note avec intérêt que les tétrasyllabes mercredisiaque et vendredisiaque fournissent à eux deux les deux tiers des attestations de dérivés de noms de jour en -iaque que nous avons trouvées sur la Toile. Dans la mesure où nous n'avons déniché qu'une quinzaine de telles attestations, on ne peut pas se fier aveuglément à cette proportion. Mais il n'est pas impossible que paradisiaque (et aphrodisiaque) pèsent sur mercredisiaque et vendredisiaque non seulement du fait de leur composition segmentale, mais aussi par leur schème tétrasyllabique. 
Le cas des dérivés de noms de jour de semaine en -ette est assez analogue à celui des dérivés en -iaque. Si l'on s'en tenait aux seules rimes riches, la sourde /s/ devrait à tout le moins concurrencer la sonore / $\mathrm{z} /$ comme consonne épenthétique, puisque Lexique 3 suggère que les noms en /-set/ sont au moins aussi nombreux que les noms en /-zet/ dans le lexique mémorisé. Le tableau 1 permet de constater qu'il n'en est rien : nous n'avons aucun cas d'insertion de la sourde devant -ette, c'est la sifflante sonore /z/ qui, parmi les dentales, seconde seule - mais peu souvent - la consonne nasale comme consonne épenthétique. Quand, toutefois, on s'intéresse aux voyelles qui précèdent les finales /-set/ et /-zet/, on constate qu'il n'y a dans Lexique 3 que deux noms en /iset/ contre dix en /izet/ (dont disette). Cette circonstance entre certainement dans l'explication du fait que /z/ soit une meilleure consonne épenthétique que /s/ devant -ette. Cuissette et pissette ne font pas le poids face à chemisette, grisette, nuisette ou disette.

\section{Les sous-séries : aspects syntaxiques}

Dans la section précédente, nous ne nous sommes fondés, pour estimer la fréquence des différentes consonnes dans le rôle de consonne épenthétique, que sur leur présence ou leur absence après le nom de chacun des jours de semaine dans nos données. Cette approximation grossière ne suffit pas dans le cas du suffixe -ien. Une analyse plus fine montre que, dans son cas, le choix de la consonne épenthétique dépend en partie de la catégorie syntaxique du dérivé.

Nous avons relevé systématiquement (octobre 2011) toutes les occurrences des dérivés de noms de jour en -nien, -sien et -tien (ou -cien, ou -ssien) sur Google en français, en nous efforçant d'éliminer les doublons et sans tenir compte des rares cas où la catégorie est indécidable :

\begin{tabular}{|c|c|c|c|c|c|c|}
\hline & \multicolumn{2}{|c|}{ /Xdinjẽ / } & \multicolumn{2}{|c|}{ /Xdizjẽ / } & \multicolumn{2}{|c|}{ /Xdisjê $\tilde{\varepsilon} /$} \\
\hline Adjectifs & 20 & $(65 \%)$ & 65 & $(44 \%)$ & 37 & $(35 \%)$ \\
\hline Noms & 11 & $(35 \%)$ & 84 & $(56 \%)$ & 68 & $(65 \%)$ \\
\hline Total : & 31 & & 149 & & 105 & \\
\hline
\end{tabular}

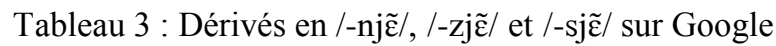

On constate que la consonne /n/ apparaît de préférence dans des adjectifs $(65 \%$ des occurrences de dérivés en -nien ont un emploi adjectival), alors que /s/ est présent de préférence dans des noms $(65 \%$ d'emplois nominaux); tel est aussi le cas de /z/, mais de façon moins marquée. Cette distribution peut être rapprochée de celle de ces consonnes dans le lexique. Dans Lexique 3, on constate que /s/ a une distribution très particulière : abstraction faite des chuintantes, qui sont peu représentées, la consonne /s/ est la seule qui apparaisse majoritairement devant -ien dans des noms (67 dérivés $(66 \%)$ contre $35(34 \%)$ pour les adjectifs) ; toutes les autres finales ou presque apparaissent majoritairement dans des adjectifs, ce qui est attendu puisque les adjectifs sont sensiblement plus nombreux que les noms.

\begin{tabular}{|l|r|r|r|r|r|r|r|r|r|r|r|r|r|r|r|r|r|l|}
\hline & $\mathrm{t}$ & $\mathrm{d}$ & $\mathrm{s}$ & $\mathrm{z}$ & $\mathrm{n}$ & $\mathrm{l}$ & $\mathrm{r}$ & $\mathrm{p}$ & $\mathrm{b}$ & $\mathrm{f}$ & $\mathrm{v}$ & $\mathrm{m}$ & $\mathrm{k}$ & $\mathrm{g}$ & $\mathrm{j}$ & 3 & $\mathrm{j}$ & $\mathrm{j}$ \\
\hline Adj. & 7 & 28 & 35 & 20 & 50 & 33 & 46 & 8 & 8 & & 7 & 9 & 4 & 1 & 1 & 7 & \\
\hline Noms & 6 & 15 & 67 & 11 & 25 & 18 & 35 & 2 & 5 & & 3 & 5 & 1 & 1 & 4 & 8 & \\
\hline
\end{tabular}

Tableau 4 : Attaques simples devant -ien dans Lexique 3 
Cette répartition n'a rien de mystérieux : le suffixe -ien sert essentiellement à former des adjectifs (qui peuvent être nominalisés); il ne sert guère à former des noms (qui peuvent être adjectivisés) que sur des noms de discipline en -ique, dont la finale est assibilée (phonétique $\rightarrow$ phonéticien). C'est avant tout l'abondance des noms de spécialiste en -icien (plus d'une trentaine dans Lexique 3) qui explique la prédominance des noms parmi les mots en /sjẽ / ; leur apport conforte la sous-série déjà abondante des noms en /-isj $\tilde{\varepsilon} /$ comme tahitien, milicien ou patricien : il y a en tout 48 noms en /isj $\tilde{\varepsilon} /$ dans Lexique 3 , alors qu'il n'y a que 17 adjectifs qui se terminent de cette façon.

Cette circonstance a toutes chances d'expliquer la prédominance marquée des noms parmi les dérivés adoptant la consonne épenthétique /s/ dans notre corpus. La configuration complexe /isjã / associée à la catégorie syntaxique Nom constitue un schème des mieux représentés dans le lexique. Si les éléments constitutifs d'une représentation impliquent la présence des uns des autres et si la récurrence d'un schème dans le lexique a un effet cumulatif, on comprend que les noms en /i _ j $\tilde{\varepsilon} /$ soient attirés dans la sous-série des noms en /isje/.

\section{Les sous-séries : aspects sémantiques}

Dans tous les cas examinés jusqu'à présent, la consonne épenthétique était une occlusive ou une fricative dentale. Il semble bien que la phonologie tende à restreindre le choix des consonnes épenthétiques à cette classe de consonnes. Il faut des circonstances très particulières pour que soient choisies des consonnes non dentales comme /p/ (dans lundipette, etc.) ou $/ \mathrm{k} /$ (dans lundical, etc.), ou encore la liquide $/ \mathrm{r} /$ dans les dérivés en -aie mentionnés plus haut. Dans le même ordre d'idées, on sait (cf. Plénat \& Roché 2004) que le français interfixe parfois une syllabe entre la base et le suffixe lorsque, associées ou non, des contraintes de taille et/ou des contraintes dissimilatives le requièrent. On observe néanmoins des interfixations des séquences /nik/ (dans lundinical, etc.) et / $\mathrm{sin} /$ (dans lundicinal, etc.) dans les dérivés de noms de jour de semaine en -al, alors que ni l'une ni l'autre de ces conditions n'est remplie. Il faut, là aussi, que des circonstances très particulières interviennent. Dans l'un et l'autre cas, l'insertion fait entrer le dérivé dans une sous-série caractérisée non seulement par sa finale, mais aussi, la plupart du temps, par son sémantisme.

Les dérivés de noms de jour en -pette ont un sens très particulier. Nous les avons trouvés chez une jeune femme intervenant régulièrement dans un forum féminin pour désigner ses interlocutrices obligées comme elle-même de garder le lit pour éviter un accouchement prématuré. Les interlocutrices du lundi étaient des lundipettes, celles du mardi des mardipettes, etc.

$$
\begin{aligned}
& \text { :hello: les samedipettes! } \\
& \text { forum.doctissimo.fr/grossesse.../delirent-mapettes-sujet_3461_227.ht... }
\end{aligned}
$$

Il s'agit donc là d'un mode de formation très idiosyncratique. Mais ces appellations ne suscitaient aucun commentaire particulier de ses correspondantes, qui acceptaient sans broncher cette finale disgracieuse.

L'emploi du suffixe -ette pour désigner des êtres féminins jeunes et sympathiques est courant. Il s'agit là d'une sous-série sémantique très fournie des dérivés en -ette. Mais pourquoi cet emploi d'une consonne très marquée comme $/ \mathrm{p} /$ là où l'on s'attendrait à un $/ \mathrm{n} /$ (cf. midinette, chipinette) ou, à la rigueur, à une sifflante ? Le mystère n'est pas grand. Dans les forums féminins consacrés à la grossesse, deux dérivés s'achevant par -pette ont vu le jour pour désigner les patientes sous le coup d'une Menace d'Accouchement Prématuré obligées de passer la journée allongées sur le canapé : on trouve des centaines d'attestations de MAPette et de canapette.

Moi ki ai été mapette et canapette, j'avais interdiction de koi ke ce soit...jusqu'à aujourd'hui www.magrossesse.com/forum/...de.../sexualite-dune-mapette-t68771

-pette est ainsi devenu, sur les forums en question, une finale associée à ce type de patientes, une soussous-série particulière d'une sous-série sémantique particulière des dérivés en -ette. Vu le contexte 
d'apparition des dérivés de noms de jour en -pette et le sens de ces dérivés, on ne peut avoir aucun doute sur le fait que le choix de la consonne épenthétique, qui fait entrer le dérivé dans cette sous-sous-série, résulte de la prégnance de ce schème.

Les très nombreux dérivés de noms de jour en -ical ont une origine certainement très semblable. Nous avons évoqué plus haut la possibilité que l'abondance insolite des dérivés en -idien soit liée à l'existence de quotidien. Nul doute que le / $/ \mathrm{k}$ / épenthétique de lundical, mardical, etc. ne provienne de la finale du seul adjectif de relation d'un nom de jour attesté dans les dictionnaires : dominical. On trouve de très nombreux témoignages du fait que cet adjectif sert de matrice à la sous-série :

En gros un foot dominical ou "samedical" suivant les possibilités du plus grand nombre. expatespagne.forumculture.net/t2535-foot-sur-madrid

Le brunch dominical etait cette semaine samedical; [...] arthurauxanges.blogspot.com/.../sance-de-rattrapage-spciale-cousine...

Par conséquent, aujourd'hui après un autre détour au marché samedical (ou devrais-je dire veille de dominical?) de St-Jacobs, [...]

bleuetfjord.blogspot.com/2007/02/exprimentation-culinaire.html

Dans Lexique 3, les dérivés en -cal - et parmi eux les dérivés en -ical (23 sur 43) - sont nombreux. Ce grand nombre peut jouer un rôle dans la création des dérivés en -cal des noms de jour. De la même façon que l'abondance des dérivés en -resque et en -lesque a pu jouer un rôle dans celle des quelques dérivés de noms de jour en -resque et -lesque qu'on trouve çà et là. Toutefois le / $/ \mathrm{k} / \mathrm{est}$, en dehors des occlusives et des fricatives dentales, la seule consonne épenthétique qui soit très abondamment attestée dans nos données. On ne peut pas ne pas rapprocher cette fréquence inhabituelle de l'existence du modèle que constitue dominical. Dans ce lexème le /k/ est « impliqué » non seulement par le contexte phonétique /i $\mathrm{al} /$, mais aussi par le sémantisme 'nom de jour' ; c'est cet aspect sémantique du schème qui explique que celui-ci soit assez prégnant pour s'imposer si souvent dans les dérivés de noms de jour ouvrable. Il compense l'obstacle que constitue le caractère marqué de $/ \mathrm{k} /$.

L'importance de l'aspect sémantique du conditionnement dans le choix des éléments « insérés » est encore plus manifeste dans des cas où l'« insertion » n'obéit pas à un conditionnement phonologique. On trouve à l'occasion dimanchical, quand dimanchal - qui est attesté aussi - satisfait les contraintes phonologiques ${ }^{7}$.

\section{Bises dimanchicales www.lepost.fr/perso/mamiza/reactions-sur-posts/170/}

On trouve même, en plus grand nombre, des dérivés comme lundinical, mardinical, mercredinical, etc., alors qu'aucune contrainte phonologique ne peut expliquer l'interfixation de /nik/ au lieu de l'insertion d'une simple consonne :

Mon sourire lundinical pour accompagner votre journée. fr.answers.yahoo.com >... > Toutes les catégories > Éducation > Futilités

Quand tu seras revenu de ta promenade mardinicale dans les bois [...] www.gayattitude.com/html/forums/.../kekecustomisation-de-profil/

Vous me connaissez moi et ce foutu stress «vendredinical» community.weightwatchers.fr/Blogs/ViewPost.aspx?threadID...

S'il en était besoin, on trouverait un argument supplémentaire plaidant en faveur de l'influence de dominical dans la répartition de la finale -inical. Les dérivés en -inical sont proportionnellement plus nombreux lorsque leur base est dissyllabique. Pour lundi, mardi, jeudi et samedi, nous trouvons dans nos données entre $20 \%$ et $40 \%$ de dérivés en -inical parmi les dérivés en -cal, alors que pour mercredi et vendredi on ne trouve qu'entre $5 \%$ et environ $10 \%$ de tels dérivés (cf. tableau 5 ). Cette répartition permet de penser que, satisfait par la simple adjonction de -cal dans le cas des bases de trois syllabes, le schème tétrasyllabique de dominical tend à imposer la syllabe supplémentaire $n i$ dans celui des bases de deux 
syllabes. Il n'est pas impossible que ce même schème explique en partie l'insertion de /ik/ dans dimanchical.

\begin{tabular}{|l|cc|cc|}
\hline & -nical & & -cal & \\
\hline lundi & 19 & $(23 \%)$ & 62 & $(77 \%)$ \\
\hline mardi & 24 & $(36 \%)$ & 42 & $(64 \%)$ \\
\hline jeudi & 13 & $(23 \%)$ & 44 & $(77 \%)$ \\
\hline samedi & 106 & $(39 \%)$ & 169 & $(61 \%)$ \\
\hline mercredi & 11 & $(11 \%)$ & 85 & $(89 \%)$ \\
\hline vendredi & 6 & $(6 \%)$ & 99 & $(94 \%)$ \\
\hline
\end{tabular}

Tableau 5 : Dérivés en -ical et en -inical sur Google

Il va presque de soi que si dominical sert ainsi de modèle, c'est parce qu'il est lui-même un dérivé de nom de jour, en fait le seul dérivé adjectival de ce type présent dans le lexique mémorisé. Dans dominical, le sémantisme 'nom de jour' associé au suffixe -al «implique»/ik/ (dimanchical) et même /inik/ (lundinical), de la même façon que la catégorie Nom associée à la séquence $i$ ien implique /s/ (lundicien) ou que la séquence phonique di_iaque implique /z/ (lundisiaque).

Un argument en sens contraire pourrait être tiré du fait que la position interfixale peut être occupée non seulement par la séquence /nik/, mais aussi par la séquence /sin/. Parmi nos données figurent une bonne vingtaine d'attestations de dérivés de noms de jour en -icinal comme celle-ci :

Et pour finir le diaporama saturnal (ou samedicinal ?), la fin du bouquet de tulipes pour concurrencer FMR.

frivoli.canalblog.com > Messages février 2008

Ces attestations - de provenances diverses - paraissent trop nombreuses pour qu'on en fasse de simples métathèses graphiques fautives de dérivés en -inical. Il est plus que probable que ces dérivés subissent l'influence de médicinal, le plus fréquent des deux adjectifs en -icinal présents dans Lexique 3 (l'autre est vicinal). Ce qui pourrait laisser penser que l'interfixation d'une séquence après les noms de jour n'obéit pas toujours à un conditionnement sémantique.

Cette conclusion toutefois nous laisse un peu sceptiques, dans la mesure où même pour l'insertion d'une simple consonne marquée comme /p/ ou / $/$ / ce type de conditionnement semble nécessaire : l'existence d'oedipien n'autorise apparemment pas la création de lundipien, mardipien, etc. Nous croyons plutôt à un scénario complexe où dominical joue un rôle. La finale -cinal apparaît principalement, comme la finale -nical, après les noms de jour de semaine dissyllabiques. Lundicinal, mardicinal, jeudicinal et samedicinal fournissent les trois quarts des attestations dans nos données ; mercredicinal - qui commence comme médicinal - est attesté plusieurs fois, mais nous n'avons qu'une attestation de vendredicinal. On peut voir là l'influence du schème tétrasyllabique de médicinal, mais il n'est pas exclu que le schème prégnant soit celui de dominical. Dans un cas comme samedicinal, par exemple, on peut supposer que ce schème appelle une syllabe supplémentaire devant le suffixe -al (/samdiCVCal/), et que le contexte /diCVCal/ appelle à son tour la séquence /sin/ qui figure dans médicinal, comme le contexte /diCjak/ appelle la consonne /z/ de aphrodisiaque et paradisiaque ${ }^{8}$. Il ne semble guère possible de démontrer la justesse de ce scénario hypothétique à partir des données dont nous disposons, mais il présente l'avantage de conforter l'idée que l'insertion d'éléments phonologiquement marqués est uniformément corrélée, dans les dérivés de noms de jour, à l'agrégation du dérivé dans une sous-série sémantique. 


\section{Conclusion}

Cette étude d'une infime province de l'épenthèse présuffixale en français montre que le conditionnement de ce phénomène est très complexe. Sa raison d'être la plus fréquente nous paraît assez simple : il s'agit de fournir à un lexème qui en est encore dépourvu un thème caractérisé, comme la majorité des thèmes présuffixaux, par une consonne finale. Mais il arrive aussi que l'insertion résulte de l'attraction exercée par une famille ou une série de dérivés particulières (cf. tintamardesque ou plouctitude). Nos analyses ont porté avant tout sur le conditionnement du choix de la consonne intercalée, et ce choix s'est révélé d'une grande complexité, en ce qu'il comporte à la fois des aspects purement phonologiques et des aspects lexicaux eux-mêmes pluriels. Pour ce qui est de la phonologie, nous avons suggéré que les épenthèses réduplicatives sont sans doute mieux représentées qu'on ne le croit en français (cf. vendredidesque) et que, de son côté, le recours à une consonne non marquée doit tenir compte des contraintes dissimilatives (cf. *vendreditette). Du point de vue lexical, enfin, l'insertion de la consonne intercalaire résulte souvent de l'attraction d'une sous-série suffixale qui, d'un point de vue phonologique peut faire intervenir à la fois une séquence de phonèmes et un schème prosodique récurrents (cf. vendredisiaque), et qui est caractérisée parfois aussi par la catégorie syntaxique dont relève le dérivé (cf. vendreditien) ou par le sémantisme de celui-ci (cf. vendredical). Il arrive ainsi qu'un lexème singulier ou qu'une toute petite famille de lexèmes impose la consonne choisie (cf. vendredipette). Le rôle des pressions lexicales sur le choix de la consonne intercalaire avait été mis en lumière par les études antérieures qui, depuis au moins Nyrop (op. cit.), faisaient abondamment intervenir les « analogies proportionnelles », mais le nombre et la diversité des cas de figure représentés dans ce minuscule fragment de lexique que constituent les dérivés de noms de jour est particulièrement frappant.

\section{Références}

Burzio, L. (2002). Surface-to-surface Morphology: When Your Representations Turn into Constraints. In P. Boucher (ed.), Many Morphologies, Somerville, Mass. : Cascadilla Press, 142-177.

Frei, H. (1929). La grammaire des fautes : introduction à la linguistique fonctionnelle. Paris : Geuthner - Genève : Kundig - Leipzig: Harrassowitz.

Kitto, C., \& P. de Lacy (1999). A correspondence theory of epenthetic quality. In C. Kitto and C. Smallwood (eds.), Proceedings of AFLA (Austronesian Formal Linguistics Association) VI [= Toronto Working Papers in Linguistics, 16.2], 181-200.

Lombardi, L. (2002). Coronal epenthesis and markedness. Phonology, 19-2, 219-251.

McCarthy, J. J., \& A. Prince (2001 [1993]). Prosodic Morphology: Constraint Interaction and Satisfaction. ROA 482.

McCarthy, J. J., \& A. Taub (1992). Compte-rendu de C. Paradis \& J.-F. Prunet, dir. (1991). Phonology, 9-2, 363-370.

Mir Marashi, F. (2005). Le suffixe -itude en français. Mémoire de DEA, Université Toulouse 2.

Nyrop, K. (1908). Grammaire historique de la langue française, t. III. Copenhague: Gyldendalske Boghandel Nordisk Forlag.

Pagliano, C. (2003). L'épenthèse consonantique en français. Ce que la syntaxe, la sémantique et la morphologie peuvent faire à la phonologie : parles-en de ta numérotation ?impossible. Thèse de doctorat, Université de Nice Sophia-Antipolis.

Pagliano, C. (2005). Élaboration d'un corpus morphophonologique : l'épenthèse consonantique à la frontière suffixale en français. Corpus [En ligne], 3 décembre 2004, mis en ligne le 2 décembre 2005, consulté le 25 novembre 2011. URL : http://corpus.revues.org/index257.html.

Paradis, C., \& J.-F. Prunet, dir. (1991). The Special Status of Coronals: Internal and External Evidence. San Diego : Academic Press.

Pichon, E. (1942). Les principes de la suffixation en français. Paris : d'Artrey. 
Plénat, M. (1999). Morphophonologie des dérivés argotiques en -ingue et en -if. Remarques sur quelques épenthèses de consonne après consonne en français. Probus, 11, 101-132.

Plénat, M. (2005). Rosinette, cousinette, starlinette, chipinette. Décalage, infixation et épenthèse devant -ette. In I. Choï-Jonin, M. Bras, A. Dagnac \& M. Rouquier (éds), Questions de classification en linguistique : méthodes et descriptions. Mélanges offerts au Professeur Christian Molinier, Berne : Peter Lang, 275-298.

Plénat, M. (2009). Le conditionnement de l'allomorphie radicale en français. Mémoires de la Société de Linguistique de Paris, Nouvelle série $n^{\circ}$ 17, 119-140.

Plénat, M. (à paraître). Dissimilatory phenomena in French word-formation. In P. O. Müller, I. Ohnheiser, S. Olsen \& Fr. Rainer (eds.), Word-Formation. An International Handbook of the Languages of Europe, Berlin - New York : De Gruyter Mouton

Plénat, M., \& M. Roché (2004). Entre morphologie et phonologie : la suffixation décalée. Lexique, 16, 159-198.

Porquier, R. (1993). Remarques sur la dérivation en -t- en français contemporain. Travaux de linguistique et de philologie, XXXI, 367-384.

Prince, A., \& P. Smolensky (2002 [1993]). Optimality Theory: Constraint Interaction in Generative Grammar. ROA 537.

Roché, M. (2009). Pour une morphologie lexicale. Mémoires de la Société de Linguistique de Paris, Nouvelle série $n^{\circ} 17,65-87$.

Roché, M. (2010). Base, thème, radical. Recherches Linguistiques de Vincennes, 39, 95-133.

Roché, M. (2011). Pression lexicale et contraintes phonologiques dans la dérivation en -aie du français. Linguistica (Ljubljana) 51, 5-22.

Stein, G. (1971). La dérivation française et le problème des consonnes intercalaires. Cahiers de Lexicologie, 18, 4364.

\footnotetext{
${ }^{1}$ Si l'on en croit Google, par exemple, la lundite aiguë (33 occurrences trouvées le 18/10/2011) est sensiblement plus fréquente que la vendredite aiguë (23 occurrences), mais ces deux affections l'emportent de beaucoup sur la mardite, la mercredite, la jeudite, la samedite et la dimanchite aiguës (entre 0 et 7 occurrences chacune), et ce sont les deux seules qui semblent évoluer en lundiose et en vendrediose. Autre exemple : il se trouve que seuls MERCREDI, SAMEDI et DIMANCHE - les jours de congé scolaire - servent de base à des dérivés en -ade désignant des tournois hebdomadaires (mercrediades, samediades, dimanchades).

${ }^{2}$ Si nous comprenons bien, Stein (art. cit.: 54) voit quant à lui dans la consonne intercalaire un moyen d'aligner la fin du radical avec une frontière de syllabe.

${ }^{3}$ Cf. les suffixes argotiques -ingue et -if, qui sont régulièrement précédés d'une coronale au besoin épenthétique (dégourdi $\rightarrow$ dégourdingue, lourd $\rightarrow$ lourdingue, louf $\rightarrow$ loufdingue; morceau $\rightarrow$ morcif, paquet $\rightarrow$ paxif $;$, voir Plénat 1999).

${ }^{4}$ Cette hypothèse prédit que l'on devrait trouver d'autres exemples d'épenthèses réduplicatives dans des dérivés dont les bases comportent déjà une répétition. On en trouve en effet, et en nombre non négligeable : dans nos données nous avons par exemple 5 attestations de ali(-)bababesque, 1 de barbapapapesque, 1 de bonobobesque (contre 12 ali(-)babatesque, 9 barbapapatesque ou barbe-à-papatesque, et 0 bonobotesque). Ces pourcentages nous semblent trop élevés pour qu'on puisse considérer les épenthèses réduplicatives comme de simples bégayages graphiques. Dans ces formes, la transgression de la contrainte dissimilative est d'autant mieux assumée que le contexte lui-même est transgressif: le locuteur a conscience d'écrire un mot «qui n'existe pas ». L'exemple des dérivés de Ali Baba et de barbe-à-papa suggère même que la répétition du même phonème puisse être délibérément recherchée.

${ }^{5}$ Nous avons testé toutes les formes fléchies de chaque suffixe, sauf en ce qui concerne -ETTE, dont nous n'avons pas pris en compte le masculin.

${ }^{6}$ En fait, bien qu'attestés après les six noms de jour, les dérivés en /-zet/ ne concurrencent pas sérieusement les dérivés en /-net/, présents en beaucoup plus grand nombre sur la Toile.
} 
${ }^{7}$ On trouve aussi dimanchinal, comme dans :

Voici voilà le Billet d'Humeur Sportive dimanchinal préparé par votre envoyée spéciale, toujours aussi professionnelle et impartiale.

soliblog.blog.mongenie.com/index/tag/pronostic

Il n'est pas impossible que cette forme doive son interfixe /in/ à machinal.

${ }^{8}$ La seule autre séquence en CVC figurant entre /di/ et /al/ dans Lexique 3 est /3it/ (dans digital). Il se trouve que nous avons aussi dans nos données quelques occurrences de jeudigital et de vendredigital, comme dans :

Cette conférence qui aurait du ouvrir le cycle les "jeudigitaux" le 14 octobre 2010, sera finalement et exceptionnellement un "vendredigital".

www.bibliotheques93.fr/?page id $=191$ \&event id $=189$

Le cas de ces formes est différent de celui des dérivés en -dicinal. Il s'agit très probablement de mots-valises, puisque ces termes s'appliquent à un cycle de conférences hebdomadaires sur le Web 2.0 proposées en fait sous le nom de Jeudigitales. 